\title{
TLR5 involvement in attenuated IL-8 production in nuclear decorin silenced oral mucosal dysplastic keratinocytes and squamous cell carcinoma*
}

\author{
Nyla Dil ${ }^{1,2 \#}$, Abhijit G. Banerjee ${ }^{1,3}$ \\ ${ }^{1}$ Department of Oral Biology, Faculty of Dentistry, University of Manitoba, Winnipeg, Canada \\ ${ }^{2}$ Department of Immunology, and Department of Medical Microbiology and Infectious Diseases, Faculty of Medicine, University of \\ Manitoba, Winnipeg, Canada \\ ${ }^{3}$ Center for Genomic Bio-Medicine and Research Institute, Durg, Chhattisgarh, India \\ Email: ${ }^{\#}$ dil@cc.umanitoba.ca
}

Received 7 August 2013; revised 3 September 2013; accepted 27 September 2013

Copyright (C) 2013 Nyla Dil, Abhijit G. Banerjee. This is an open access article distributed under the Creative Commons Attribution License, which permits unrestricted use, distribution, and reproduction in any medium, provided the original work is properly cited.

\begin{abstract}
Head and neck cancer is one of the most prevalent cancers in the world. Roughly half of these malignancies originate from oral mucosa and constitute oral squamous cell carcinomas. Despite many advances in diagnostic and therapeutic regimens, five-year survival rate remains at roughly $50 \%$, indicating the need for in depth understanding of the oral squamous cell carcinoma immunobiology. We have previously shown that in human dysplastic oral keratinocytes (DOK) and malignant squamous cell carcinoma (SCC-25), multifunctional proteoglycan decorin is aberrantly expressed and localized in the nucleus bound to nuclear EGFR. In vitro nuclear decorin knockdown significantly reduced IL-8 and IL8-dependent migration, invasion and angiogenesis in these cells. Since toll-like receptor (TLR) signalling leads to IL-8 production, we examined here if these receptors played a role in decorin silencing mediated reduction in IL-8 levels in oral mucosal dysplastic keratinocytes and squamous carcinoma cells. Decorin silenced DOK and SCC-25 cells showed a marked diminution of TLR5 mRNA and protein expression compared with respective controls that translated into the loss of function in response to appropriate TLR ligand. In these mucosal oral epithelia, decorin stable knockdown significantly down-regulated IL-8 production following activation with TLR5 ligand flagellin. These data suggest that decorin silencing interferes with IL-8 production, in part, by altering TLR5 expression and signaling in dysplastic and malignant oral epithelia.

\footnotetext{
${ }^{*}$ Competing Interests: No financial or non-financial competing interests exist.

${ }^{\#}$ Corresponding author.
}

This study highlights the significance of TLR5 expression and signaling and its plausible interactions with proteoglycans in mucosal cancers.

Keywords: Oral Cancer; Mucosal Cancer; Nuclear Decorin; TLR5; IL-8; Flagellin; PAMP; DAMP; SLRP; Proteoglycans

\section{BACKGROUND}

Head and neck squamous cell carcinoma (HNSCC) is a devastating disease that accounts for roughly 1.6 million newly diagnosed cases and 330,000 deaths each year worldwide [1,2]; half of these cancers are localized in the oral cavity and are termed oral squamous cell carcinoma. A multifactorial mucosal cancer, oral squamous cell carcinoma, is primarily associated with chronic tobacco, alcohol use and betel chewing; however, chronic inflammation, viral infections (human papillomavirus), genetic predisposition and poor oral hygiene have also been strongly implicated in oral tumorigenesis [3-5].

The majority of inflammatory conditions are triggered by Toll-like receptors (TLRs) which are a large family of at least 11 evolutionarily conserved proteins characterized by ligand specific activation and complex downstream signaling [6-8]. TLRs are predominantly expressed on various immune and structural cells where they serve as innate cell surface pathogen sensors and promote local inflammation by triggering the induction of various proinflammatory mediators, including IL-8. Recently, TLR expression or up-regulation has been detected in various tumour types, especially in epithelium derived cancers [9-11]. Expression of TLRs varies in different cancerous cell types; however, evidence indi- 
cates that TLR expression is functionally associated with tumorigenesis. It has been suggested that TLR expression might promote malignant transformation of epithelial cells $[11,12]$. Engagement of TLRs promotes tumor development and metastasis, protects the cancerous cells from immune attack and induces resistance to apoptosis and chemo-resistance in some malignancies [10,12-14].

In addition to its putative proinflammatory functions, IL-8 is a potent angiogenic factor and plays an important role in cancer progression and metastasis [15-18]. IL-8 is responsible for most of the angiogenic activity induced by human oral mucosal dysplastic and malignant epithelial cells $[19,20]$. Previously, we have shown that in human dysplastic oral keratinocytes (DOK) and malignant squamous carcinoma cells (SCC-25), multifunctional proteoglycan decorin is atypically expressed and localized in the nucleus bound to nuclear epidermal growth factor receptor (EGFR) [18,21]. Post-transcriptional nuclear decorin knock-down resulted in significantly reduced IL-8 and IL8-dependent migration, invasion and angiogenic potential in these dysplastic and malignant oral mucosal epithelia $[18,20]$. Since TLR signaling is an upstream event in the majority of cytokines/chemokine expression, in this current study, we examined if TLR pathway is implicated in the aberrant nuclear decorin silencing mediated attenuated IL-8 production.

\section{MATERIALS AND METHODS}

\subsection{Cell Lines and Culture Conditions}

Oral mucosa origin, premalignant-Dysplastic Oral Keratinocytes (DOK) and malignant-Squamous Carcinoma (SCC-25) cell lines were routinely maintained in DMEM/F12 (Hyclone, Logan, Utah) supplemented with $10 \%$ Foetal Calf Serum for use as in vitro model in our studies, as described previously [20,22,23]. Silencing of decorin gene expression was achieved using short hairpin RNA (shRNA) technology as described earlier [18]. Briefly, oligonucleotides targeting decorin transcript variants $\mathrm{A} 1$ and $\mathrm{A} 2$ and scrambled sequence control were custom synthesized, annealed, and cloned into shRNA expression vector pGeneClip Puro ${ }^{\mathrm{TM}}$ (Promega) by Super Array Bioscience Corporation (Frederick, MD). BLAST queries were performed to ensure that the sequences have no significant homology with any other human genes. Transformation grade shRNAi plasmids were transfected into DOK and SCC-25 cells using Effectene ${ }^{\mathrm{TM}}$ transfection reagent following manufacturer's protocol (Qiagen, Valencia, CA). The stable transfectants were selected for puromycin (Calbiochem, San Diego, CA) resistance at $2.5 \mu \mathrm{g} / \mathrm{ml}$ optimal concentration. Pools of stable transfectants (maintained at $1 \mu \mathrm{g} / \mathrm{ml}$ of puromycin) were used in all experiments to avoid clone-specific differences. Decorin knock down was confirmed at transcript and protein level by quantitative real-time reverse transcription-PCR and Western blotting, respectively. Pooled decorin-shRNA transfected DOK or SCC-25 clones showed more than $80 \%$ reduction in decorin mRNA expression and almost complete abrogation of decorin protein expression in nuclear lysates and/or in whole cell lysates when compared to control-shRNA transfected clones or no transfection wild type DOK [18]. Herein, untransfected DOK and SCC-25 cells will be referred to as wild type (WT), scrambled shRNA stable transfectants as control (or Ctrl-shRNA in figures), and decorin shRNA stable transfectants as decorin silenced (or DCNshRNA in figures).

\subsection{Multiplex PCR}

The transcript expression levels of innate immune TLRs and co-regulatory molecules were quantified in decorin silenced, control, and WT DOK and SCC25 cells using multiplex PCR (MPCR) kit for human signaling receptor set-2 (TLR1, TLR2, TLR3, TLR5, TLR6, TLR9 and CD14) from Maxim Biotech, San Francisco, CA. This set also included housekeeping gene-GAPDH, as internal cDNA loading control in each reaction. MPCR was carried out according to the manufacturer's instructions. Briefly, 1X MPCR buffer, 2.5 units of Taq DNA polymerase, and cDNA template from DOK and SCC25 cells were mixed in a $25 \mu \mathrm{l}$ reaction and subjected to 35 cycles of PCR, with denaturing, annealing, and extension temperatures at $96^{\circ} \mathrm{C}, 67^{\circ} \mathrm{C}$, and $70^{\circ} \mathrm{C}$, respectively. Following MPCR, the DNA amplicons were fractionated electrophoretically on $2 \%$ agarose gel containing $0.5 \mu \mathrm{g} / \mathrm{ml}$ ethidium bromide.

\subsection{Real-Time PCR}

RNA was extracted from DOK and SCC-25 cells using RNeasy Plus mini kit (Qiagen, Valencia, CA) and $2.5 \mu \mathrm{g}$ of total RNA was used to synthesize cDNA, using Suprescript III Reverse Transcriptase (Invitrogen, San Diego, CA). Quantitative RT-PCR was performed using QuantiTect $^{\mathrm{TM}}$ SYBR Green PCR kit (Qiagen, Valencia, CA) on the Mini Opticon ${ }^{\mathrm{TM}}$ Real-Time PCR system (BioRad, Hercules, CA) as per manufacturer's protocol. Quantitative PCR primer pairs were designed for SYBR Green chemistry based detection of amplicons for TLR5 (5'-TGCATTAAGGGGACTAAGCCT-3', 5'-AAAAGGGAGAACTTTAGGGACT-3') and GAPDH (5'-ACAGTCAGCCGCATCTTCTT-3', 5'-GTTAAAAGCAGCCCT G GTGA-3'). GAPDH was used as relative house-keeping gene expression control to normalize for sample variations.

\subsection{Western Blot Analysis}

Cells were rinsed with ice-cold PBS and were lysed in a 
buffer containing $20 \mathrm{mM}$ Tris, $\mathrm{pH} 7.6,0.1 \%$ SDS, $1 \%$ Triton-X, $1 \%$ deoxycholate, $100 \mu \mathrm{g} / \mathrm{ml}$ PMSF, and protease inhibitor cocktail (Sigma-Aldrich, St. Louis, MO). Lysates were centrifuged at $20,000 \mathrm{xg}$ for $20 \mathrm{~min}$ at $4^{\circ} \mathrm{C}$. Protein concentration was determined by Bis-Cinchonic Acid (BCA) protein assay (Pierce, Rockford, IL) and subjected to $10 \%$ SDS-PAGE analysis, followed by transfer to polyvinylidene difluoride membrane (Bio-Rad, Hercules, CA). The membranes were immunoprobed with 1:500 dilution of monoclonal antibody to human TLR5 (Alexis Biochemicals, San Diego, CA) or 1:1000 dilution of anti-human beta-tubulin polyclonal antibody. Western blots were developed with appropriate horseradish peroxidase conjugated secondary antibodies (Bio-Rad) and ECL Plus chemiluminescence system (Amersham, Arlington Heights, IL) and exposed to auto radiographic films. Radiographs were scanned and densitometry analysis was done using AlphaEase FC software (Alpha Innotech Corporation, San Leandro, CA).

\subsection{ELISA for IL-8 Quantification}

Decorin silenced, control and WT DOK and SCC-25 cells $\left(5 \times 10^{5}\right.$ cells $/$ well $)$ were cultured in complete medium in 24-well flat-bottom plates at a final volume of a $500 \mu$ l. Cells were stimulated with varying concentrations of flagellin (Alexis Biochemicals, San Diego, CA); $100 \mathrm{ng} / \mathrm{ml}$ concentration was found to be optimal. Culture supernatants were collected after $24 \mathrm{~h}, 48 \mathrm{~h}$ or $72 \mathrm{~h}$ of incubation and IL-8 was assayed with $100 \mu \mathrm{l}$ of cell free culture supernatant using DuoSet IL-8 ELISA kit (R \& D Systems, Minneapolis, MN) according to manufacturer's instructions. Absorbance was read at $450 \mathrm{~nm}$ with the SPECTRAMax 190 microplate spectrophotometer and results were analyzed by SOFTMax Pro software (Molecular Devices, Sunnyvale, CA). Sample concentrations were determined by interpolation from the standard curve. IL-8 lower detection limit was found to be 5.6 $\mathrm{pg} / \mathrm{ml}$. Samples were read in triplicate.

\subsection{Statistical Analysis}

Student's paired $t$ test was used to determine the statistical significance of the data. Statistical analysis was performed on Graph Pad Prism Software. Significance was evaluated at $p$ values:

$$
{ }^{*} p<0.05,{ }^{* *} p<0.01,{ }^{* * *} p<0.001 \text {. }
$$

\section{RESULTS}

\subsection{TLR5 Expression down Regulation in Decorin Silenced DOK and SCC25 Cells}

Toll-like receptor expression has been described in many cancers especially epithelial derived tumours and has been linked to tumour progression [24]. Many of the
TLRs are known to trigger IL-8 expression. We sought to determine whether nuclear decorin silencing has an effect on any or all of the TLRs expression in dysplastic and malignant oral epithelial cells. Multiplex PCR analysis showed that out of an array of TLRs, TLR5 expression was significantly reduced in decorin silenced DOK and SCC-25 cells compared to respective WT and control cells (Figure 1). Interestingly, no difference was observed in the expression of TLR1, TLR2, TLR3, TLR6, TLR9 and CD14 between decorin silenced and WT/control DOK or SCC-25 cells (Figure 1). We confirmed this observation by Real time PCR analysis using specific primers; no difference was observed in the expression of TLR1, TLR2, TLR3, TLR4, TLR6, TLR8 and TLR9 between decorin silenced and WT or controls dysplastic or malignant epithelial cells (data not shown). However, Real time PCR analysis using TLR5 specific primers showed more than $75 \%$ reduction in TLR5 expression in decorin silenced DOK and SCC-25 cells (Figure 2(a)). To investigate if this reduction in TLR5 expression translates into lower TLR5 receptor levels, we performed Western blot analysis and observed similar TLR5 protein reduction in decorin silenced DOK and SCC- 25 cells in comparison to TLR5 protein expression in respective WT and/or control cells (Figures 2(b) and (c)).

\subsection{Attenuated Flagellin-Induced IL-8 Production in Decorin Silenced DOK and SCC25 Cells}

We have shown previously that nuclear decorin silencing results in attenuated constitutive IL-8 production in oral mucosal DOK and SCC-25 cells. Here, we sought to determine if nuclear decorin silencing-mediated TLR5 down regulation has an effect on IL-8 induction in these dysplastic and malignant oral epithelial cells. Flagellin is a known ligand for TLR5 and flagellin stimulation of

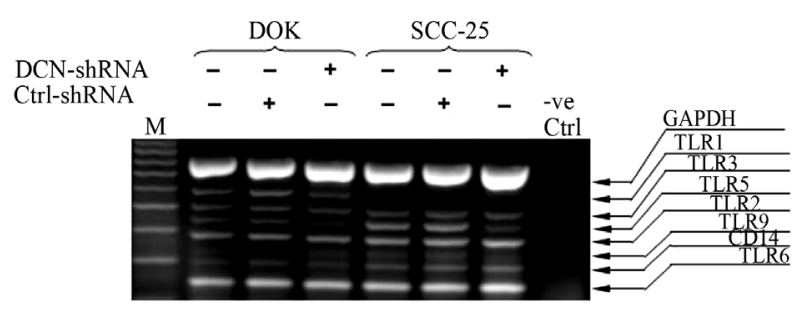

Figure 1. Toll-like receptors and CD14 expression in decorin silenced and WT dysplastic and malignant oral epithelial cells. DOK and SCC-25 cells were stably transfected with decorinshRNA (DCN-shRNA), or scrambled sequence-shRNA (CtrlshRNA) or no transfection control (WT). RNA was extracted and cDNA was subjected to multiplex RT-PCR to detect multiple pattern recognition receptor transcripts. MPCR products were fractionated electrophoretically on a $2 \%$ agarose gel containing $0.5 \mathrm{mg} / \mathrm{ml}$ ethidium bromide, visualized under UV light and photographed. The results shown are representative of three independent experiments. 


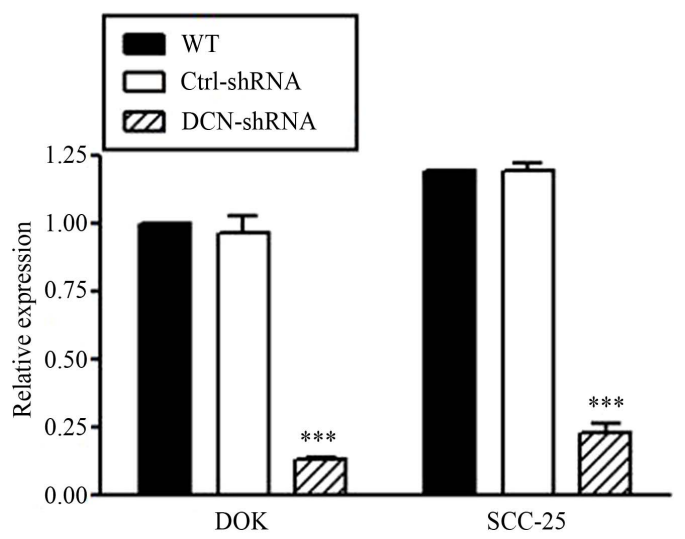

(a)

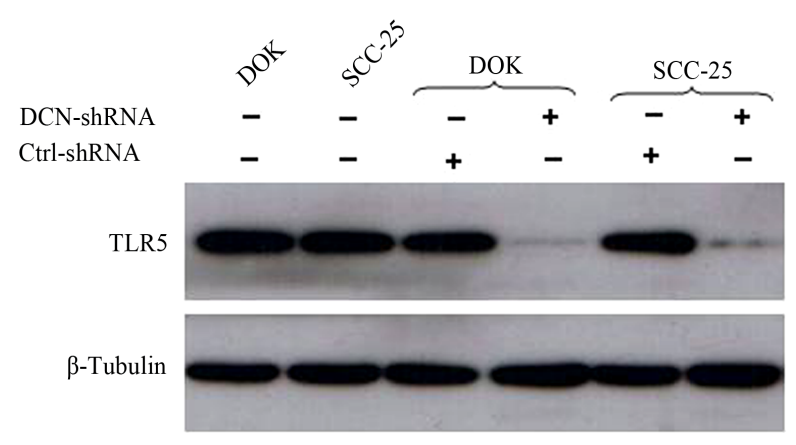

(b)

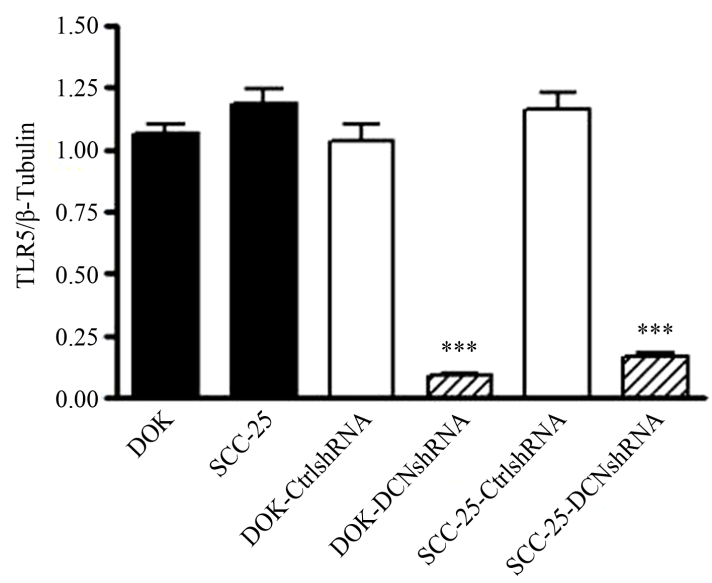

(c)

Figure 2. TLR5 down regulation in decorin silenced DOK and SCC25 cells. RNA was extracted from WT, control and decorin silenced DOK and SCC-25 cells and (a) cDNA was subjected to quantitative RT-PCR, normalized TLR5 expression from one representative experiment of three. (b) Cell lysates were collected as described in materials and methods and subjected to SDS-PAGE followed by immunoblotting using anti-TLR5 and antitubulin antibodies. (c) Densitometric analysis is presented as a histogram of TLR5 relative band density from 3 experiments. ${ }^{* * *} p<0.001$ compared to respective controls.

epithelial cells results in increased IL- 8 production. To investigate the TLR5 link in IL-8 mitigation in decorin silenced dysplastic and malignant epithelia, we deter- mined and compared the levels of IL-8 production upon flagellin stimulation in these cells. Briefly, cells were stimulated with flagellin for 24,48 and $72 \mathrm{~h} ; 24 \mathrm{~h}$ time point was considered optimal for IL-8 production. Consistent with down regulation of TLR5 expression levels, we found a significant reduction in flagellin stimulated IL-8 production in decorin silenced cells compared to WT or ctrl-shRNA treated DOK or SCC-25 cells (Figure 3). It is interesting to note that SCC-25 cells produce much higher levels of flagellin stimulated IL-8 compared to dysplastic oral keratinocytes.

\section{DISCUSSION}

A number of functions have been assigned to small leucine-rich proteoglycan (SLRPs) decorin. The most intriguing function is the ability to inhibit growth and metastasis of a range of tumors. Accordingly, decorin is being studied extensively as a naturally occurring potential oncosuppressive agent [25]. Decorin is normally present in the extracellular stromal compartment and analyses of various tumors indicate that it is rarely expressed by cancer cells. However, studies by us and others indicate that there are exceptions to this prevailing decorin expression and decorin mediated cancer growth and suppression model [21,26,27]. Ectopic decorin expression has been linked to increased vascular endothelial growth factor expression and increased angiogenesis [28]. In our previous studies of oral precancerous and cancerous lesions and cellular models of oral cancer progression, we demonstrated that decorin is aberrantly expressed and localized in the nucleus bound to nuclear epidermal growth factor receptor (EGFR) in the dysplastic and ma-

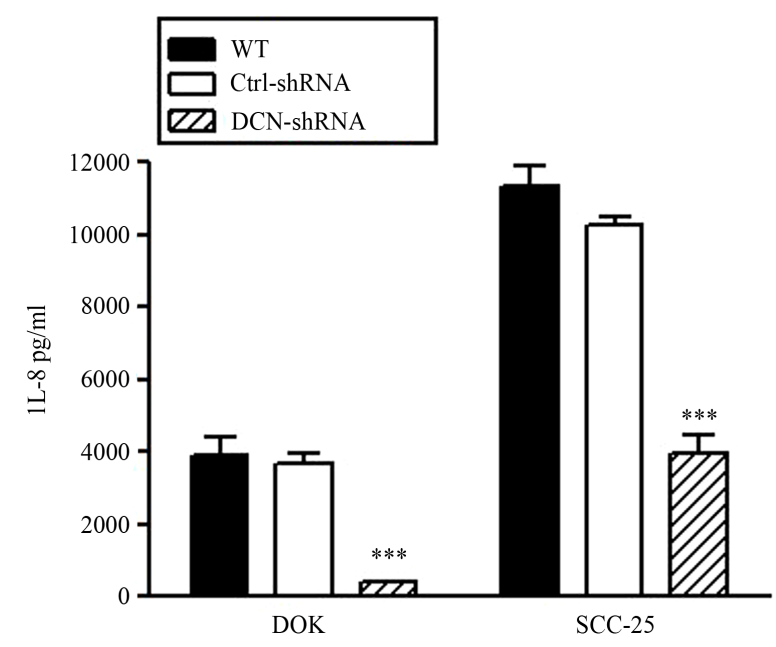

Figure 3. Reduced Flagellin-induced IL-8 production in decorin Silenced DOK and SCC25. Cells were cultured with $100 \mathrm{ng} / \mathrm{ml}$ flagellin and IL-8 was measured in $24 \mathrm{~h}$ culture supernatants using ELISA. Data are presented as mean $\pm \mathrm{SD}$ of three replicates of one representative experiment of four. ${ }^{* * *} p<0.001$ compared to respective controls. 
lignant oral epithelial cells $[18,21]$. Stable knock down of nuclear decorin resulted in significant down regulation of IL-8 and IL-8 dependent migration, invasion and angiogenic potential in this oral cancer cell line model. Here, we show that reduced IL-8 in nuclear decorin silenced DOK and SCC-25 cells is mediated, in part, by reduced TLR5 expression and signaling.

Toll-like receptors are a major component of an evolutionarily conserved, classic pattern recognition system, which underpins pathogen recognition, mediates inflammatory responses and bridges innate and adaptive immunity. Historically, TLR agonists have been used as adjuvant in anti cancer immunotherapy. However, a growing body of clinical and experimental studies suggest that the neoplastic process may actually subvert TLR signalling pathway to enhance cancer progression. Hence, the TLR system appears to play the role of a double-edged sword in the neoplastic development.

Increased expression of various TLRs has been found to be associated with a variety of cancers of gastrointestinal, oral and genital mucosa $[11,29]$. Of all the TLRs, TLR5 is the most prominent TLR that appears to have a role in mucosal neoplastic processes. Enhanced TLR5 expression has been reported in gastric and colorectal malignancies, as well as in precancerous lesions of uterine cervix $[30,31]$. Stimulation with TLR5-ligand, flagellin, increased proliferation and induced TLR5 mediated IL-8 production in various gastric cancer cell lines [32]. A role for TLR5 has also been described in mucosal head and neck cancers. In biopsy sample of 119 oral tongue squamous cell carcinoma patients, TLR5 expression was found to be more abundant and widespread in cancer cells than in the adjacent healthy lingual epithelial tissue [33]. In this oral cancer cohort, TLR5 expression levels predicted prognosis in terms of recurrence and patient survival. In salivary gland adenocarcinoma, flagellin activation of TLR5 promoted migration and invasion of cancer cells [12]. Hence, increased TLR5 expression is correlated with mucosal cancer progression and, therefore, has been proposed as a biomarker for gastric and cervical dysplasia as well as oral cancer and might help identify dysplastic lesions in mucosal epithelium $[30,31$, 33].

The canonical function of proteoglycans is the maintenance and regulation of the architecture of various extracellular matrices (ECM). Decorin is an important structural component of the ECM and a fundamental regulator of collagen fibrillogenesis that got its eponym based on its ability to decorate collagen fibrils. However, a growing body of recent evidence indicates that secreted proteoglycans in particular those from the small leucinerich family (including decorin), hyaluronan-binding gene family as well as the glycosaminoglycan hyaluronan act as endogenous ligand for TLR2, TLR4 and TLR6 in ad- dition to their above mentioned widely recognized structural function $[34,35]$. These proteoglycans and other endogenous molecules act as "danger signals" and can activate pattern recognition receptors and thus are referred to as damage-associated molecular patterns (DAMPs) [36]. Any direct or indirect interaction between decorin and TLR5 has hitherto not been described. We show here for the first time that under-expressing aberrant nuclear localized decorin results in down regulation of TLR5 and TLR5 mediated IL-8 production in dysplastic and malignant oral epithelial cells. This might be due to nuclear decorin interactions with its binding partner nuclear EGFR (which acts as a transcription factor) or additional interactions with other nuclear and/or cytosolic factors. Further studies are warranted to decipher these interactions and to explore how exactly the gene expression of TLR5 is regulated by nuclear localization of decorin in these cells.

\section{CONCLUSION}

Using shRNA expression plasmid mediated in vitro stable nuclear decorin silencing, we present here a study of the involvement of TLRs in attenuated IL- 8 production upon nuclear decorin knockdown in dysplastic and malignant oral mucosal epithelial cells. We demonstrate that nuclear decorin knockdown significantly inhibits TLR5 (a putative mucosal cancer biomarker) and TLR5 mediated IL- 8 production in these cells. These findings and our previous work on nuclear decorin suggest that nuclear decorin silencing suppresses major tumor development and progression processes such as IL-8 dependent migration, invasion, and angiogenesis in part by mitigating the expression of TLR5. This study also highlights the significance of TLR5 expression and signaling in development and progression of mucosal cancers. It might be potentially useful in developing a therapeutic regimen for patients presenting with premalignant or malignant oral mucosal lesion accompanied with poor oral health associated mucosal inflammation.

\section{AUTHORS' CONTRIBUTIONS}

ND contributed to study concept, performed, and analyzed the experiments, interpreted data and wrote the manuscript. AGB conceptualized nuclear decorin study and designed and BLAST decorin targeting oligonucleotides, analyzed results and interpreted data, and approved the final manuscript and directed the research program. All authors reviewed and approved the final manuscript.

\section{ACKNOWLEDGEMENTS}

The authors gratefully acknowledge Dr. J.K. Vishwanatha, University of North Texas, Fort Worth for providing the oral cancer progression model cell lines and Linda Delmage, Charlton Cooper, Susan Pylypas, 
University of Manitoba for technical assistance. This project was supported by research grants form Manitoba Health Research Council and Manitoba Medical Service Foundation and by funds from Department of Oral Biology and Faculty of Dentistry, University of Manitoba.

\section{REFERENCES}

[1] Jemal, A., Siegel, R., Ward, E., Hao, Y.P., Xu J.Q., Murray, T. and Thun, M.J. (2008) Cancer statistics, 2008. CA: A Cancer Journal for Clinicians, 58, 71-96. http://dx.doi.org/10.3322/CA.2007.0010

[2] Jemal, A., Center, M.M., Ward, E. and Thun, M.J. (2009) Cancer occurrence. Methods in Molecular Biology, 471, 3-29. http://dx.doi.org/10.1007/978-1-59745-416-2 1

[3] Parkin, D.M., Bray, F., Ferlay, J. and Pisani, P. (2005) Global cancer statistics, 2002. CA: A Cancer Journal for Clinicians, 55, 74-108.

http://dx.doi.org/10.3322/canjclin.55.2.74

[4] Dobrossy, L. (2005) Epidemiology of head and neck cancer: Magnitude of the problem. Cancer and Metastasis Reviews, 24, 9-17.

http://dx.doi.org/10.1007/s10555-005-5044-4

[5] Ramqvist, T. and Dalianis, T. (2010) Oropharyngeal cancer epidemic and human papillomavirus. Emerging Infectious Diseases, 16, 1671-1677. http://dx.doi.org/10.3201/eid1611.100452

[6] Medzhitov, R., Preston-Hurlburt, P. and Janeway, Jr. C.A. (1997) A human homologue of the Drosophila Toll protein signals activation of adaptive immunity. Nature, $\mathbf{3 8 8}$, 394-397. http://dx.doi.org/10.1038/41131

[7] Takeda, K., Kaisho, T. and Akira, S. (2003) Toll-like receptors. Annual Review of Immunology, 21, 335-376. http://dx.doi.org/10.1146/annurev.immunol.21.120601.14 $\underline{1126}$

[8] Akira, S., Uematsu, S. and Takeuchi, O. (2006) Pathogen recognition and innate immunity. Cell, 124, 783-801. http://dx.doi.org/10.1016/i.cell.2006.02.015

[9] Furrie, E., et al. (2005) Toll-like receptors-2, -3 and -4 expression patterns on human colon and their regulation by mucosal-associated bacteria. Immunology, 115, 565574. http://dx.doi.org/10.1111/j.1365-2567.2005.02200.x

[10] Kelly, M.G., et al. (2006) TLR-4 signaling promotes tumor growth and paclitaxel chemoresistance in ovarian cancer. Cancer Research, 66, 3859-3868. http://dx.doi.org/10.1158/0008-5472.CAN-05-3948

[11] Lee, J.W., et al. (2007) Increased toll-like receptor 9 expression in cervical neoplasia. Molecular Carcinogenesis, 46, 941-947. http://dx.doi.org/10.1002/mc.20325

[12] Park, J.H., et al. (2011) Toll-like receptor 5 activation promotes migration and invasion of salivary gland adenocarcinoma. Journal of Oral Pathology \& Medicine, 40, 187-193.

http://dx.doi.org/10.1111/j.1600-0714.2010.00929.x

[13] He, W., et al. (2007) TLR4 signaling promotes immune escape of human lung cancer cells by inducing immunosuppressive cytokines and apoptosis resistance. Molecular Immunology, 44, 2850-2859. http://dx.doi.org/10.1016/j.molimm.2007.01.022

[14] Droemann, D., et al. (2005) Human lung cancer cells express functionally active Toll-like receptor 9. Respiratory Research, 6, 1. http://dx.doi.org/10.1186/1465-9921-6-1

[15] Koch, A.E., et al. (1992) Interleukin-8 as a macrophage-derived mediator of angiogenesis. Science, 258, 1798-801. http://dx.doi.org/10.1126/science.1281554

[16] Strieter, R.M., et al. (1995) Role of C-X-C chemokines as regulators of angiogenesis in lung cancer. Journal of Leukocyte Biology, 57, 752-762.

[17] Murdoch, C., et al. (1999) Cxc chemokine receptor expression on human endothelial cells. Cytokine, 11, 704-712. http://dx.doi.org/10.1006/cyto.1998.0465

[18] Dil, N. and Banerjee, A.G. (2011) A role for aberrantly expressed nuclear localized decorin in migration and invasion of dysplastic and malignant oral epithelial cells. Head \& Neck Oncology, 3, 44. http://dx.doi.org/10.1186/1758-3284-3-44

[19] Lalla, R.V., et al. (2001) Fibrin induces IL-8 expression from human oral squamous cell carcinoma cells. Oral Oncology, 37, 234-242. http://dx.doi.org/10.1016/S1368-8375(00)00090-7

[20] Dil, N.and Banerjee, A.G. (2012) Knockdown of aberrantly expressed nuclear localized decorin attenuates tumour angiogenesis related mediators in oral cancer progression model in vitro. Head \& Neck Oncology, 4, 11. http://dx.doi.org/10.1186/1758-3284-4-11

[21] Banerjee, A.G., et al. (2003) Aberrant expression and localization of decorin in human oral dysplasia and squamous cell carcinoma. Cancer Research, 63, 7769-7776.

[22] Hu, L., et al. (1991) Abnormal expression of retinoic acid receptors and keratin 19 by human oral and epidermal squamous cell carcinoma cell lines. Cancer Research, 51, 3972-3981.

[23] Hsu, S. et al. (2002) Transforming growth factor beta 1 dysregulation in a human oral carcinoma tumour progression model. Cell Proliferation, 35, 183-192. http://dx.doi.org/10.1046/j.1365-2184.2002.00237.x

[24] Yu, L. and Chen, S. (2008) Toll-like receptors expressed in tumor cells: Targets for therapy. Cancer Immunology, Immunotherapy, 57, 1271-1278. http://dx.doi.org/10.1007/s00262-008-0459-8

[25] Neill, T., et al. (2012) Decorin: A guardian from the matrix. American Journal of Pathology, 181, 380-387. http://dx.doi.org/10.1016/j.ajpath.2012.04.029

[26] Zafiropoulos, A., et al. (2008) Decorin-induced growth inhibition is overcome through protracted expression and activation of epidermal growth factor receptors in osteosarcoma cells. Molecular Cancer Research, 6, 785794. http://dx.doi.org/10.1158/1541-7786.MCR-07-0165

[27] Zafiropoulos, A. and Tzanakakis, G.N. (2008) Decorinmediated effects in cancer cell biology. Connective Tissue Research, 49, 244-248. http://dx.doi.org/10.1080/03008200802147746

[28] Santra, M., et al. (2008) Ectopic decorin expression up-regulates VEGF expression in mouse cerebral endothelial cells via activation of the transcription factors $\mathrm{Sp} 1$, HIF1alpha, and Stat 3. Journal of Neurochemistry, 105, 
324-337.

http://dx.doi.org/10.1111/j.1471-4159.2007.05134.x

[29] Schmausser, B., et al. (2005) Toll-like receptors TLR4, TLR5 and TLR9 on gastric carcinoma cells: An implication for interaction with Helicobacter pylori. International Journal of Medical Microbiology, 295, 179-185. http://dx.doi.org/10.1016/j.ijmm.2005.02.009

[30] Pimentel-Nunes, P., et al. (2011) Increased expression of Toll-like receptors (TLR) 2, 4 and 5 in gastric dysplasia. Pathology \& Oncology Research, 17, 677-683. http://dx.doi.org/10.1007/s12253-011-9368-9

[31] Kim, W.Y., et al. (2008) Increased expression of Tolllike receptor 5 during progression of cervical neoplasia. International Journal of Gynecological Cancer, 18, 300305. http://dx.doi.org/10.1111/j.1525-1438.2007.01008.x

[32] Song, E.J., et al. (2011) Flagellin promotes the proliferation of gastric cancer cells via the Toll-like receptor 5. International Journal of Medical Microbiology, 28, 115-
119.

[33] Kauppila, J.H., et al. (2013) Toll-like receptor 5 (TLR5) expression is a novel predictive marker for recurrence and survival in squamous cell carcinoma of the tongue. British Journal of Cancer, 108, 638-643. http://dx.doi.org/10.1038/bjc.2012.589

[34] Schaefer, L., et al. (2005) The matrix component biglycan is proinflammatory and signals through Toll-like receptors 4 and 2 in macrophages. Journal of Clinical Investigation, 115, 2223-2233.

http://dx.doi.org/10.1172/JCI23755

[35] Frey, H., et al. (2013) Biological interplay between proteoglycans and their innate immune receptors in inflammation. FEBS Journal, 280, 2165-2179.

[36] Chen, G.Y. and Nunez, G. (2010) Sterile inflammation: Sensing and reacting to damage. Nature Reviews Immunology, 10, 826-837. http://dx.doi.org/10.1038/nri2873 